\title{
Characterization of a cracked specimen with full-field measurements: direct determination of the crack tip and energy release rate calculation
}

\author{
Rostand Moutou Pitti • Claudiu Badulescu • \\ Michel Grédiac
}

Received: 30 June 2013 / Accepted: 17 December 2013 / Published online: 14 January 2014

(C) The Author(s) 2014. This article is published with open access at Springerlink.com

\begin{abstract}
The crack characterization in a pre-cracked aluminum specimen is investigated in this study using the grid method. The images of this grid are analyzed to provide the crack tip location as well as the displacement and strain fields on the surface of the specimen during a tensile test. Experimental data are used to calculate the energy release rate with the compliance method A fracture analysis is also performed using the invariant $\mathrm{M} \theta$ integral in which both real and virtual displacement fields are introduced. This integral is implemented in the finite element software Cast3M. Both approaches give similar results in 2D case.
\end{abstract}

Keywords Aluminum - Crack tip identification · Finite element analysis · Grid method $\cdot \mathrm{M} \theta$ integral

R. Moutou Pitti $(\varangle) \cdot$ M. Grédiac

Clermont Université, Université Blaise Pascal, Institut

Pascal, EA 3867, 63000 Clermont Ferrand, France

e-mail:rostand.moutou_pitti@univ-bpclermont.fr

R. Moutou Pitti · M. Grédiac

CNRS, UMR 6602, Institut Pascal, 63171 Clermont

Ferrand, France

R. Moutou Pitti

CENAREST, IRT, BP 14070, Libreville, Gabon

C. Badulescu

UENSTA Bretagne, Brest, France

e-mail:claudiu.badulescu@ensta-bretagne.fr

M. Grédiac

e-mail:Michel.Grediac@univ-bpclermont.fr

\section{Introduction}

In the mechanical and civil engineering fields, many materials such as wood, asphalt, composites, steel, polymers or aluminum are submitted to complex and long-time loadings (Moutou Pitti et al. 2008; Réthoré et al. 2010; Grédiac and Toussaint 2013). Among several cases, the real serviceability of structures made of these materials is often due to the crack initiation and the crack growth process during their lifetime (Dubois and Moutou 2012). This must be taken into account to enhance the reliability of such structures (Moutou Pitti and Chateauneuf 2012). The characterization of the crack tip location and growth appears therefore to be a key-issue in the communities involved in prediction of the behavior of structures and in machine safety (Chalivendra 2009; Atkinson and Eftaxiopoulos 1992; Suo and Combescure 1992; Parks 1974, 1977). This is due to the singularity of the mechanical fields at the crack tip on the one hand, and to the problems raised by the identification of the crack tip location and propagation on the other hand, even when recent image analysis methods are employed.

An overview of the literature in this field shows that many studies have been undertaken in order to characterize cracking in various types of materials. In the past, McNeill et al. (1987) have been already evaluated stress intensity factor by means of digital image correlation. Recently, Zanganeh et al. (2013) have localized the crack tip using a comparative study based 
on displacement field data. The digital image correlation (DIC) technique has been used in order to characterize crack tip growth (Yates et al. 2010; Pop et al. 2011). Recently, Méité et al. (2013a,b) have studied mixed-mode fracture by means of full-field optical and finite element calculations. DIC has also been applied to evaluate fatigue crack propagation law with the socalled integrated digital image correlation for which an a priori solution for the displacement/strain fields is necessary to reduce measurement noise (Mathieu et al. 2011). The same technique has been proposed to extract fracture mechanics parameters from kinematic measurements (Réthoré et al. 2012), and to identify crack tip location (Réthoré et al. 2012). Some of these methods are combined with a finite element analysis to update at the same time the actual mechanical fields during the crack propagation process. Réthore et al. (2008) have pointed out the drawback due to the virtual extension field theta that plays a major role when displacement measurements are noisy. Several authors also mention the use of interaction integrals to extract fracture mechanics parameters (Réthore et al. 2005, 2008 for instance).

In the present paper, the grid method is employed to analyze the cracking process in an aluminum specimen. The big advantage is that no a priori closed-form solution for the displacement/strain distribution is necessary to analyze the strain field, the latter being directly measured, within certain limits, at the crack tip despite the high strain gradient that occurs. This is obtained thanks to the very good compromise between strain resolution and spatial resolution of this measurement method.

The paper is organized as follows. First, the main characteristics of the grid method are recalled. The experimental device and the experimental procedure are then briefly described. Obtained results are given and discussed by comparing typical experimental and numerical strains and displacement maps. In the second section the invariant integral $M \theta$ formulation applied in the isotropic case is recalled and then used to compute the energy release rate in the opening mode case. In the third section, the procedure employed to determine the crack tip localization is described. It is directly based on some features of the strain maps near the crack tip. The crack tip location is identified for different values of the load applied to the specimen. The critical energy release rate is deduced from the measurements using the compliance method, assuming that displace- ments are imposed. These values are finally compared with the numerical energy release rate given by the $M \theta$ integral approach.

\section{Experimental procedure}

\subsection{The grid method}

The grid method is one of the white-light techniques available to measure bidimensional displacement and strain fields. It consists first in depositing a crossed grid on the surface of the specimen under investigation in order to track with a camera the slight change in the grid as loading increases. In the current case, a 12bit/1,040 × 1,376 pixel SENSICAM camera connected to its companion software CamWare was employed. The grid was transferred using the procedure described in (Piro and Grédiac 2004). The pitch of the grid was equal here to $0.2 \mathrm{~mm}$ along both directions.

With this technique, the in-plane displacement and strain fields are deduced from the images of the grid taken during the test by processing them with the windowed Fourier transform (WFT). This transform actually provides the phases and its derivatives of the quasi-regular marking of the surface. It can be easily demonstrated that the change of the phase between any current grid image and the reference grid image is directly proportional to the in-plane displacement (Surrel 2000). Strain components and local rotations are merely obtained from the spatial derivatives of the phase, using the definition of these quantities (respectively half the sum and half the difference between the displacement gradient and its transpose). In practice the WFT is merely carried out by convolving the grid images by a suitable kernel. Since the window of this transform has a certain width greater than one pixel, estimating the strain components at a given pixel relies on the information contained in the neighboring pixels. The kernel of the WFT used here to perform this calculation is a 2D Gaussian envelope whose standard deviation is equal to sigma $=5$ pixels. Using the classic "3-sigma rule", the width of this envelope can be estimated to $2 \times 3 \times 5=30$ pixels. The lowest distance between two independent measurements being equal to the width of the Gaussian envelope, this width is actually the spatial resolution of the technique for strain measurement. Note that sigma $=5$ pixels is the lowest value that can be used here because the frequency of the 
grid in the image is $\mathrm{f}=1 / 5$ pixels -1 and because the following inequality holds: $\mathrm{f} \times$ sigma $\geq 1$, as recently rigorously demonstrated in Sur and Grédiac (2014).

It has been shown that the metrological performance of this technique could be significantly improved (especially for small strain measurements) by getting rid of most of the grid marking defects that unavoidably occur when grids are printed on their support (Badulescu et al. 2009a,b) . In particular, a very good compromise is obtained between resolution in strain and spatial resolution. For instance, a typical resolution is strain is some hundreds of microstrains for a spatial resolution in strain equal to 30 pixels. These quantities however strongly depend on various parameters such as the quality of the grid and lighting. Another feature of this technique is that the displacement and strain components are calculated pixelwise, thus allowing detecting very localized phenomena. Note however that these measured quantities are not independent from one pixel to each other because measuring the phases and their derivatives is performed at any pixel by relying on the information contained in the neighboring pixels. Full details on strain calculation with this technique can be found in (Badulescu et al. 2009a,b) for unidirectional and crossed grids, respectively.

\subsection{Experimental device and procedure}

The test was performed on a pre-cracked aluminum specimen with a thickness of $10 \mathrm{~mm}$. The aluminum 2024-T3 is used in this study with the Young modulus of $70 \mathrm{GPa}$, the shear modulus of $28 \mathrm{GPa}$, the shear strength of $283 \mathrm{MPa}$, the ultimate tensile strength of $483 \mathrm{MPa}$ and the tensile yield strength of $345 \mathrm{MPa}$. The choice of this metal is due to his good machinability, surface finish capability and his high strength of adequate workability. A schematics view is shown in Fig. 1. As may be seen, a horizontal notch was first machined but the dimensions of the specimen are not those of a standard CT specimen. The reason is that it was first placed in an Arcan-like testing device which was rotated by $\theta=30^{\circ}$, thus the notch was rotated by $\theta$ with respect to the horizontal direction. This device was then mounted in a MTS servo-hydraulic fatigue testing machine and subjected to a cyclic load so that a crack appeared and progressively propagated. The test was stopped as soon as the crack was $3 \mathrm{~mm}$ in length. Hence the specimen featured at the end of this



Fig. 1 Specimen geometry and boundary condition

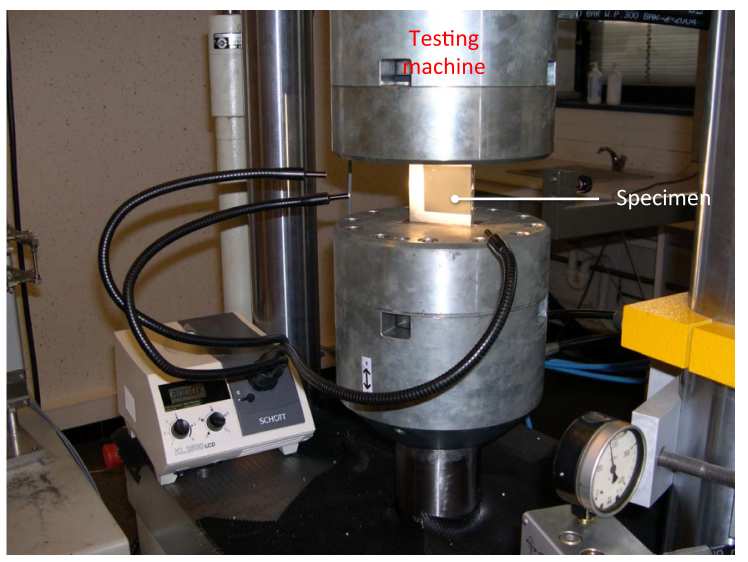

Fig. 2 Pre-cracked specimen mounted in the testing machine

first loading stage a crack inclined by $\theta$ with respect to the notch (see Fig. 1). This pre-cracked specimen was then machined so that it could be placed in the grips of a quasi-static tensile machine (see Fig. 2). A quasi-static test was finally performed up to failure.

A $48.35 \times 41.6 \mathrm{~mm}^{2}$ grid was also transferred after the fatigue test around the crack in order to measure the displacement and strain fields in this region during the quasi-static test by applying the grid image processing technique described above. 
This procedure illustrate the fact that both the location of the crack tip and the strain distribution can be obtained for various crack angles, the orientation of the crack near the tip changing during the quasi-static test to become perpendicular to the applied load.

\section{Invariant integrals}

For the simple fracture mode, the energy release rate $G$ is generally computed by the curvilinear $J$-integral (Rice 1968). Chen and Shield (1977) introduced another integral, which also takes into account mixed-mode fractures. Its main development is based on invariant integrals and conservatives laws (Noether 1971), which induce a bilinear form of the free energy density energy. Moutou Pitti et al. (2008), completed this formulation by adding a propagation term. Finally, the $M$-integral writes as follows:

$$
\begin{aligned}
\mathrm{M}= & \frac{1}{2} \int_{\Gamma_{1}}\left(\sigma_{\mathrm{ij}, 1}^{\mathrm{v}} \cdot \mathrm{u}_{\mathrm{i}}-\sigma_{\mathrm{ij}}^{\mathrm{u}} \cdot \mathrm{v}_{\mathrm{i}, \mathrm{j}}\right) \cdot \mathrm{n}_{\mathrm{j}} \mathrm{d} \Gamma_{1} \\
& +\frac{1}{2} \int_{\mathrm{V}}\left(\left(\sigma_{\mathrm{ij}}^{\mathrm{v}} \cdot\left(\mathrm{u}_{\mathrm{i}, \mathrm{j}}\right)_{, 1}+\sigma_{\mathrm{ij}}^{\mathrm{u}} \cdot\left(\mathrm{v}_{\mathrm{i}, \mathrm{j}}\right)_{, 1}\right)\right. \\
& \left.-\left(\left(\sigma_{\mathrm{ij}}^{\mathrm{v}} \cdot \mathrm{u}_{\mathrm{i}, \mathrm{j}}\right)_{, 1}+\left(\sigma_{\mathrm{ij}}^{\mathrm{u}} \cdot \mathrm{v}_{\mathrm{i}, \mathrm{j}}\right)_{, 1}\right)\right) \mathrm{dV}
\end{aligned}
$$

The first integral is the stationary crack and the second one accounts for the crack growth. This integral involves a combination of derivatives of the real displacements $u_{i}$ and stresses $\sigma_{i j}^{(u)}$ on the one hand, and derivatives of the virtual displacements $v_{i}$ and stresses $\sigma_{i j}^{(v)}$ on the other hand.

The $M$-integral in Eq. (1) involves stress components and displacement gradients. Using a finite element discretization, these fields are computed at the integration points of the mesh. In this case, the curvilinear domain $\Gamma_{1}$ requires a numerical interpolation. It is therefore preferred to transform the boundary integral into a surface integral. According to the Destuynder's technique (Destuynder et al. 1983), the integration domain is bordered by a map denoted $\vec{\theta}$ defined by the curve shown in Fig. 3. This vector must be continuous and differentiable on the domain $\Omega$ under consideration.

According to these conditions, the general form of the $M$-integral is given as follows (Moutou Pitti et al. 2008):

$$
M \theta=\frac{1}{2} \int_{\Omega}\left(\sigma_{i j}^{u} \cdot v_{i, k}-\sigma_{i j, k}^{v} \cdot u_{i}\right) \cdot \theta_{k, j} \mathrm{~d} V
$$

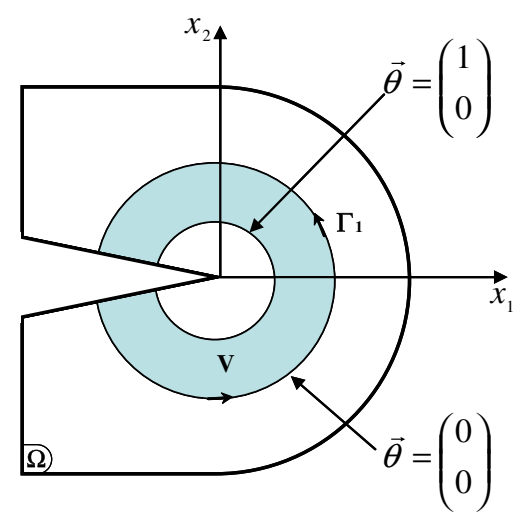

Fig. 3 Integration domain (Moutou Pitti et al. 2008)

$$
\begin{aligned}
& +\frac{1}{2} \int_{\Omega}\left(\left(\sigma_{i j}^{v} \cdot\left(u_{i, j}\right)_{, k}+\sigma_{i j}^{u} \cdot\left(v_{i, j}\right)_{, k}\right)\right. \\
& \left.-\left(\left(\sigma_{i j}^{v} \cdot u_{i, j}\right)_{, k}+\left(\sigma_{i j}^{u} \cdot v_{i, j}\right)_{, k}\right)\right) \cdot \theta_{k} \mathrm{~d} V
\end{aligned}
$$

Moutou Pitti et al. (2007), have shown that the $M \theta$ integral is equal to the energy release rate by substituting the virtual and real fields. Thus:

$G \theta=M \theta=C_{1} \cdot \frac{\left({ }^{u} K_{I}\right)^{2}}{8}$ with ${ }^{u} K_{I}=\frac{8 \cdot M \theta\left({ }^{v} K_{I}^{=} 1\right)}{C_{1}}$

$C_{1}$ is the reduced elastic compliances in mode I. ${ }^{u} K_{I}$ and ${ }^{v} K_{I}$ denote the real and virtual stress intensity factors for the opening mode, respectively. The real stress intensity factor ${ }^{u} K_{I}$ which appears in Eq. (3) can be obtained by considering a particular value for the virtual stress intensity factors, as shown in the following equation

\section{Results and discussion}

\subsection{Displacement and strain maps}

\subsubsection{Comparison between numerical and experimental results}

Numerical and experimental results are first compared in order to check that the measurement method provides reliable results. Various horizontal and vertical experimental displacement fields are shown in Fig. 4a, $\mathrm{c}$, respectively. In this figure, note that $1 \mathrm{pixel}=40 \mu \mathrm{m}$ 
(a)

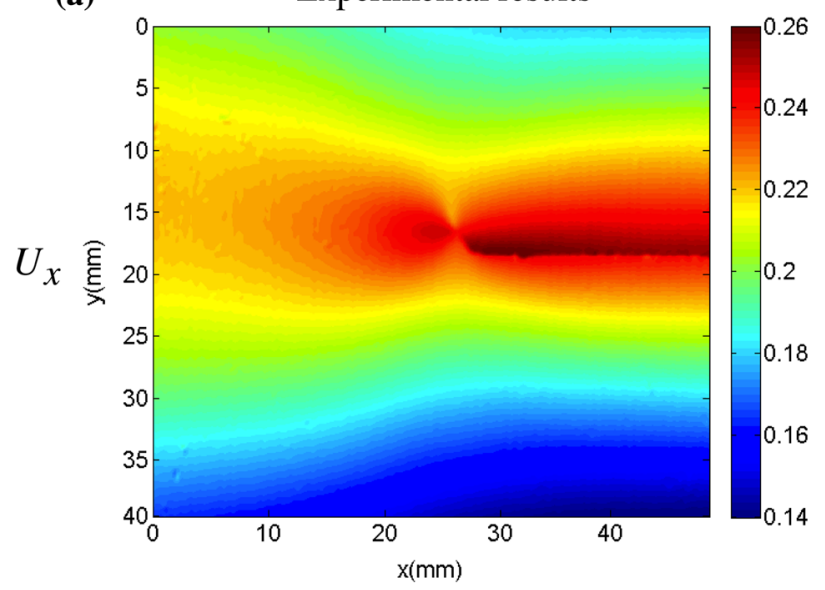

(c)

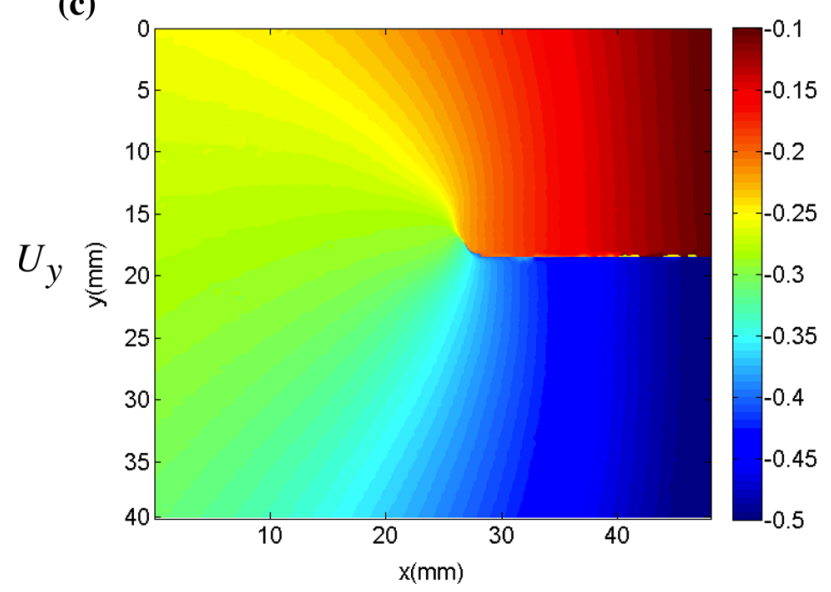

(b) Finite element results



(d)

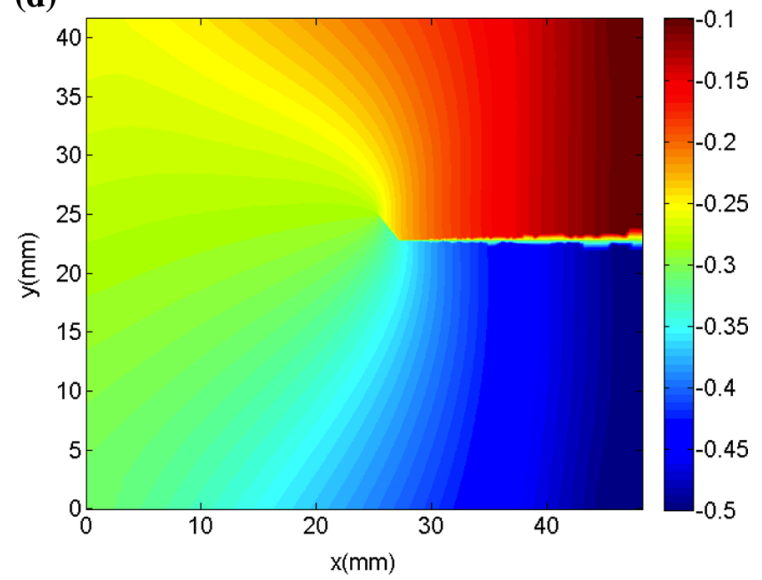

Fig. 4 Numerical and experimental displacement maps $(1$ pixel $=40 \mu \mathrm{m}), \mathrm{F}=70.95 \mathrm{kN}$

and the crack opening is given in millimeters. They are compared with their numerical counterparts obtained with a FE calculation performed with the ABAQUS Finite Element package (see Fig. 4b, d, respectively) in $2 \mathrm{D}$ case. Note that these simulations are carried out within the framework of linear elasticity, so plasticity that is likely to appear at the crack tip is not taken into account. 10500 CPS4R elements were used for the model. The constitutive material is assumed to be isotropic elastic linear, with a Young's modulus equal to $E=70 \mathrm{GPa}$ and a Poisson's ratio $v$ equal to 0.34 . The boundary conditions in this case are obtained by collecting the experimental displacement distributions at the top and bottom of the grid and imposing them to the FE model, see Fig. 5. Numerical and experimental results are globally in good agreement. On close inspection however, it can be observed that the curva- ture of the isolines located just above the crack tip is more pronounced for the numerical model. The difference is small, but it will obviously be magnified by differentiation when calculating the strain components, as confirmed below.

The corresponding experimental strain maps $\varepsilon$ are shown in Fig. 6a, c, e and compared again with the maps obtained with the FE model, see Fig. 6b, d, f. As may be seen, the very high strain gradient that occurs at the crack tip is clearly visible even though it is slightly noisy. Interestingly, some differences can be observed between numerical and experimental maps. Concerning $\varepsilon_{\mathrm{xx}}$ (see Fig. 6a, b) the blue lobes are much wider in the experimental map while the red ones are very similar. The experimental $\varepsilon_{\text {yy }}$ distribution (see Fig. 6c) exhibits bigger lobes than the numerical one (see Fig. 6d). These lobes are also more inclined in the 
Fig. 5 Finite element model and boundary conditions (a)

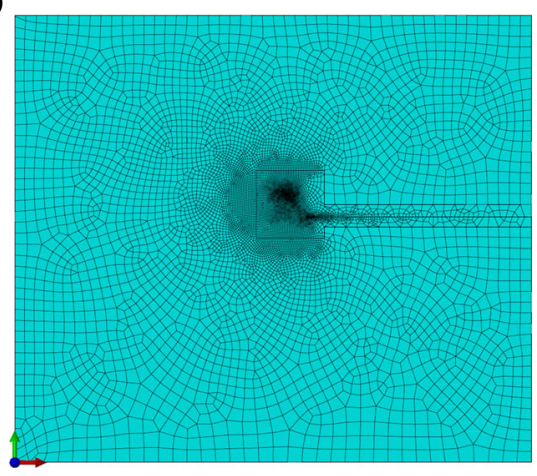

(b)

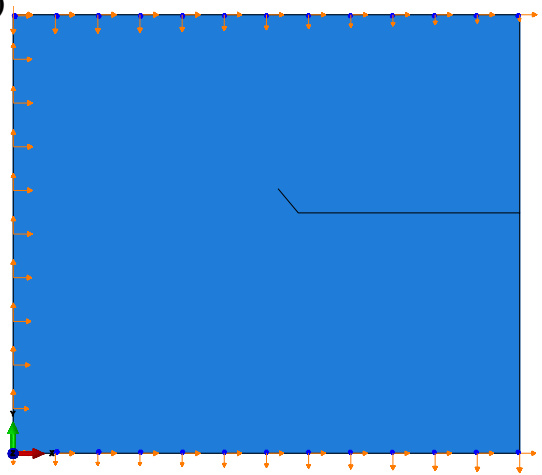

experimental map. The red lobes in the $\varepsilon_{\mathrm{xy}}$ experimental map (see Fig. 6e) are smaller than in the numerical map (see Fig. 6f). The inclination angle of the red lobe located at the top is also different between the two maps. These slight differences can potentially be due to various causes. For example, the crack tip may be not perfectly perpendicular to the front face of the specimen because 3D phenomena arise, or the actual angle of the crack near the tip is not exactly that used in the model, or a small plastic zone spans in front of the crack tip. This result emphasizes that methods based the use of an a priori knowledge of the form of the displacement and strain distributions near the crack tip may provide incorrect results if the model is not suitably chosen.

This type of strain map was used to determine the crack tip location and to deduce the crack propagation during the test, as explained in the following section.

\subsection{Finding the crack tip location from the strain and local rotation maps}

Finding the location of the crack tip is a key issue in many problems dealing with cracked specimen characterization. In many recent studies in which DIC is involved, an a priori knowledge of the form of the strain field ahead of the crack is necessary to have an information that the measurement technique cannot directly provide. In this case, the crack tip location is part of the unknowns that are measured. This location is found by performing optimization calculations (Zanganeh et al. 2013 for instance). In addition to these calculations, the problem is that any deviation of the actual strain distribution from the model, as in the example discussed above, can be really detected since no reliable measurement of the strain field in this zone can be made, the res- olution and spatial resolution in strain of the measurement method used being not sufficient. In the present study, the fact that strain fields can be measured despite the high strain gradient (beyond a certain strain level which is the resolution of the technique) leads the crack tip location to be determined directly. Finding the crack tip has been performed here using two different routes: the first one relies on the strain fields, the second one on the local rotation fields. They are briefly explained and illustrated below.

It can be seen in Fig. 6e that the $\varepsilon_{x y}$ strain field ahead of the crack tip exhibits lobes. Taking the absolute value of this quantity therefore leads to a distribution which features clear peaks and valleys (see Fig. 7). It is observed that these valleys all converge to a unique point which is actually the crack tip. In addition, the valley floor is approximately a straight line near the crossing point between the valleys. The idea is to take advantage of these two remarks by finding the valley floor in the experimental strain maps, and then to deduce the crossing point between these lines, thus giving the location of the crack tip.

This procedure was tested first on synthetic data obtained with a finite element model. This model, which contains 12500 CPS4R elements, is depicted in Fig. 5. The $\varepsilon_{\mathrm{xy}}$ strain field is depicted in Fig. 8b. This strain field is obtained first with an irregular mesh because the mesh density is greater near the crack tip. This first field is then interpolated on a regular mesh to mimic measurements that would be obtained on a regular grid: actually the CCD chip of the camera since a measurement point is available at each pixel, see Sect. 2 above. Taking the absolute value of this distribution leads to the lobes shown in Figs. 7, 8 shows the subsequent stages of the procedure for $\mathrm{F}=70.95 \mathrm{~N}$. First the valley floors are merely obtained by collecting the 

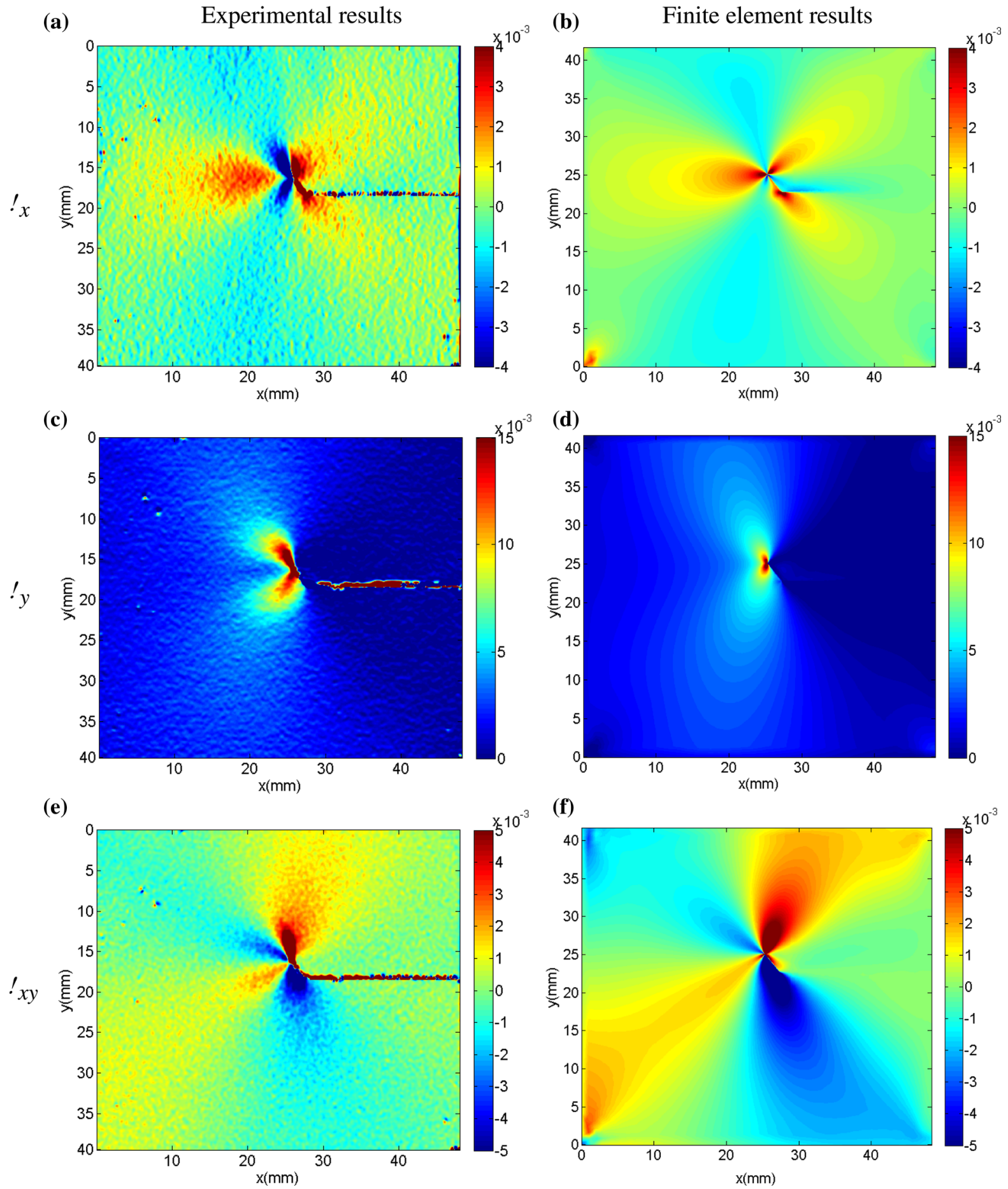

Fig. 6 Numerical and experimental strain maps, $F=70.95 \mathrm{~N}$

points for which the strain amplitude is lower than a threshold value chosen here to be equal to $1.5 \mathrm{E}-04$ (white points in the figure). This threshold value is defined by trial and error. It is slightly greater than the value that actually takes place along the valley floor to be able to find the points located along it. 


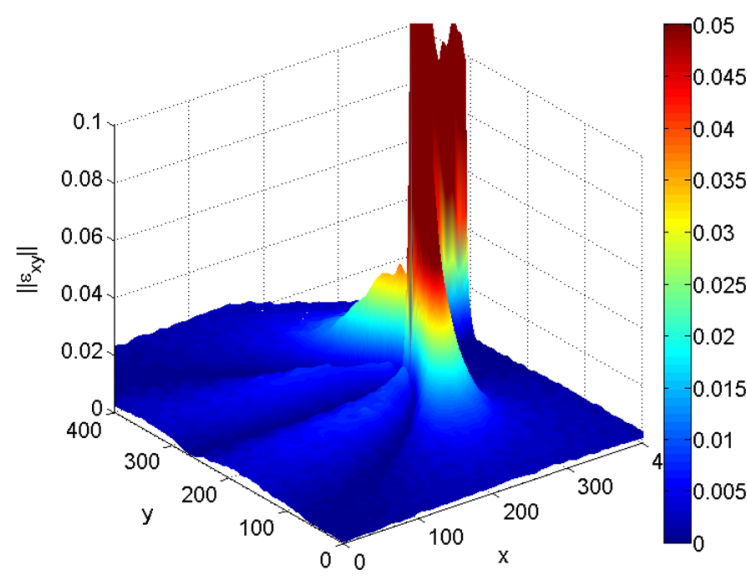

Fig. 7 3D Strain configuration

The points located in each valley and near the crack tip are then collected (red points in the figure). They are used to define a straight line for each valley by linear regression of the white point cloud. Since there are here three lines, the information is redundant and there is no unique crossing point in practice. The crack tip location is therefore considered to be the point located at the lowest distance from the three crossing points in the least square sense. Figure 8 shows such straight lines superimposed to each valley. Comparing the coordinates of the actual crack tip considered to define the mesh $(633,628)$ (or $(25.30,25.09)$ in $\mathrm{mm})$ and the identified one $(633.50,627.15)$ (or $(25.28,25.08)$ in $\mathrm{mm})$ shows that the distance between them is tiny $(0.022 \mathrm{~mm}$ in the current example).

A second simulation has been performed with a crack which becomes curved near the tip, as in the current experiment when the crack propagates, thus leading to lobes whose shape is different from the preceding one. Despite this new crack shape, performing the same procedure leads to an error of the same order of magnitude as in the preceding case (results not reported here).

Finally, the preceding examples have been reconsidered after adding a white Gaussian noise to the reference numerical fields, (see Fig. 8b). The amplitude of this noise is equal to $3.90 \mathrm{E}-04$, which is roughly the same order of magnitude as the actual noise observed in the actual strain maps processed in this study. The preceding coordinates for the identified crack tip become $(633.64,626.54)$ (or $(25.31,25.03)$ in $\mathrm{mm})$, which leads to an error of $0.286 \mathrm{~mm}$, which remains quite acceptable. Figure 9 shows the experimental location of the crack tip for different applied loads $F$ between 40 and $97.35 \mathrm{kN}$.

Another possibility for the determination of the crack tip is to consider the local rotation map. An example is shown in Fig. 10. This map features only one valley, but this valley directly points to the crack tip. The very high contrast between the colors in this very confined region helps finding the location of the crack tip directly on the map. The fact that only one valley is observed instead of three for the strain map leads to a lower resolution in the crack tip location, but both approaches are complementary. The reason is that the grid began to damage at the end of the test, when the crack went through the specimen. This made it difficult to use the first method because the lobes were not all clearly visible. The second method was therefore employed from $\mathrm{F}=97.35 \mathrm{kN}$ on. Typical local rotation maps are shown in Fig. 11. It can be seen that the deep blue valley goes to the crack tip while the effect of the damage of the grid appears mainly at the top of the crack. Both methods have been applied
Fig. 8 Crack type identification with and without noise (a)

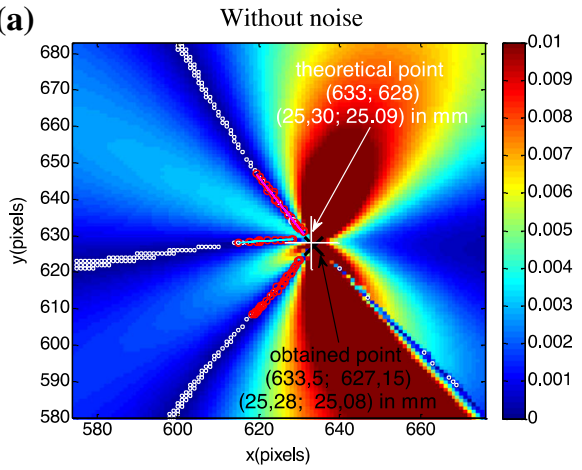

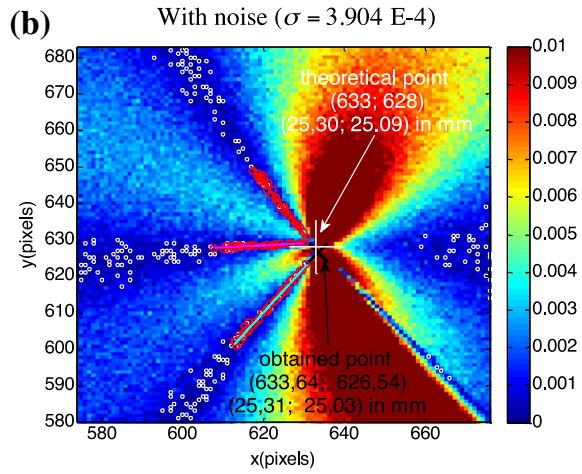


Fig. 9 Close-up view of the strain field near the crack tip. Straight lines provide the location of the crack tip for various loading levels
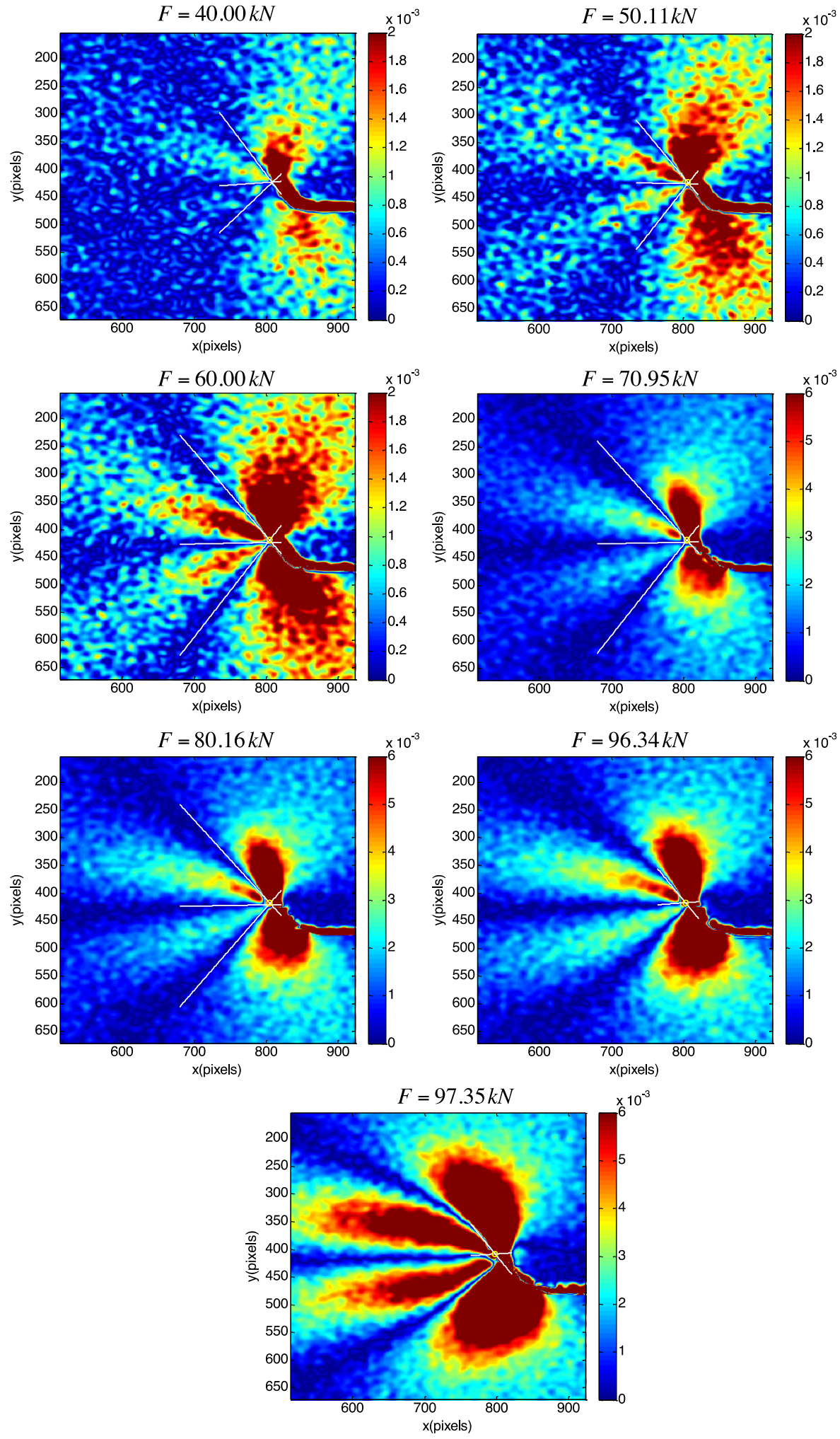
Fig. 10 Local rotation maps
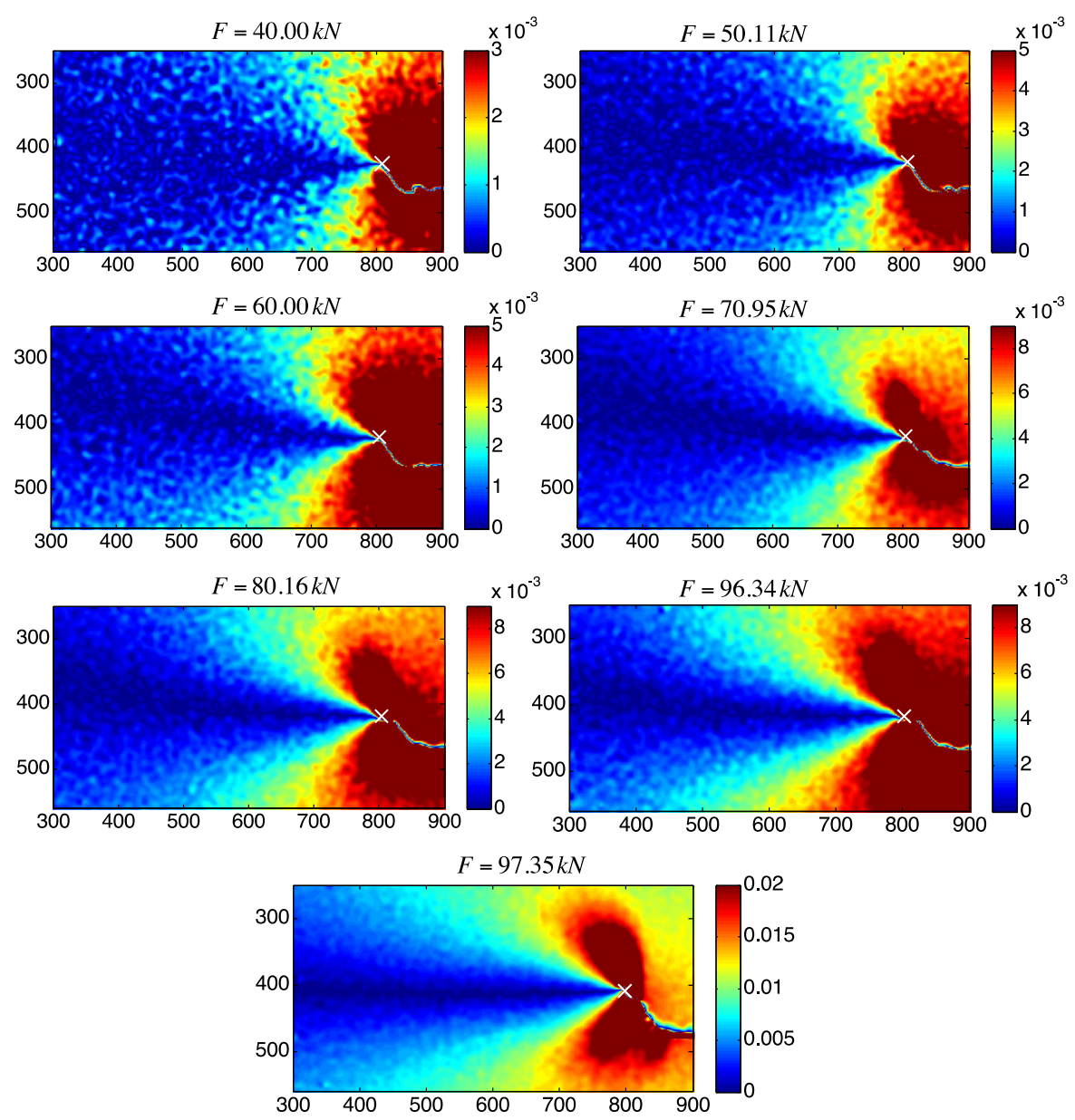

to the experimental measurements obtained here with the grid method. Obtained results are gathered in Fig. 9 where the lines used to determine the crack tip are superimposed to the strain field when the first method is employed (see thin white portions of lines). The resulting crack tip coordinates are reported in Table 1. In this table, $F$ corresponding the critical force providing crack propagation.

As a last remark, noise impairing the current strain and local rotation measurements can be seen in Figs. 9 and 10 (see the blobs superimposed to the smooth actual strain and rotation fields, especially for low values of the applied load). The relative weight of this noise logically decreases as the strain level increases from one figure to each other. It is however worth noting that noise does not seem to impair the crack tip location for the early stages of the load since the three straight lines converge to nearly the same point.
Note that the out-of-plane movement induces fictitious strain whose order of amplitude is equal to $\Delta l / l$, where 1 is the distance between the grid and the CCD chip of the camera (Molimard and Surrel 2012), and $\Delta l$ the amplitude of the movement. This distance being equal to $80 \mathrm{~cm}$ and the amplitude of this movement being no greater than $1 \mathrm{~mm}$, this fictitious strain is not greater than $0.0125 \%$, which is negligible compared to the strain values that are provided by the system in this zone.

\subsection{Crack tip location}

Figure 12 presents the evolution of the load versus the applied displacement, and Fig. 13 the same evolution versus time. The corresponding locations are recalled in Table 1. 

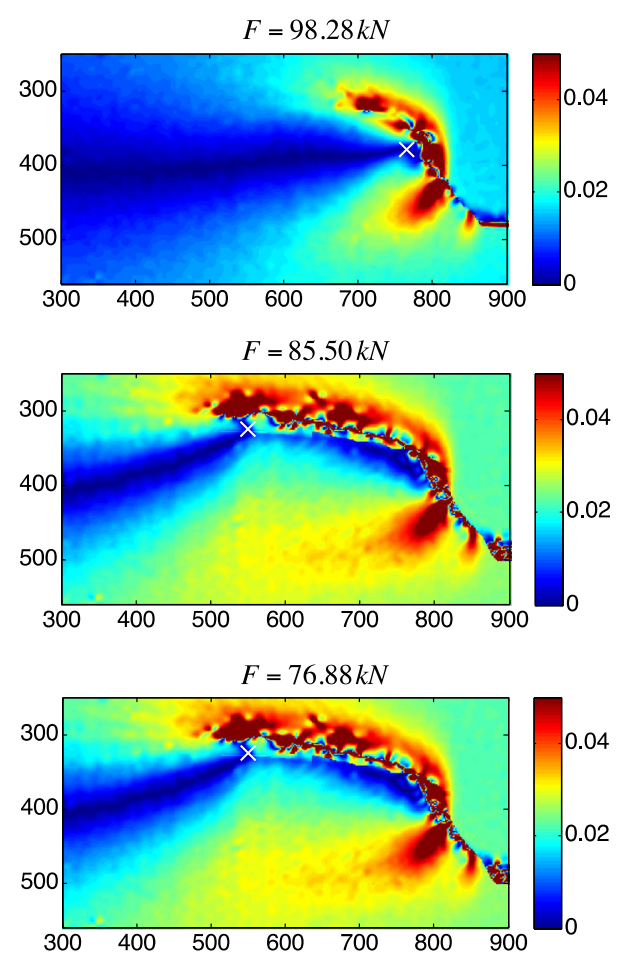

Fig. 11 Local rotation maps at the end of the test

\subsection{Critical energy release rate}

The experimental evaluation of the critical energy release rate can be performed by using the compliance method with an imposed displacement. This quantity

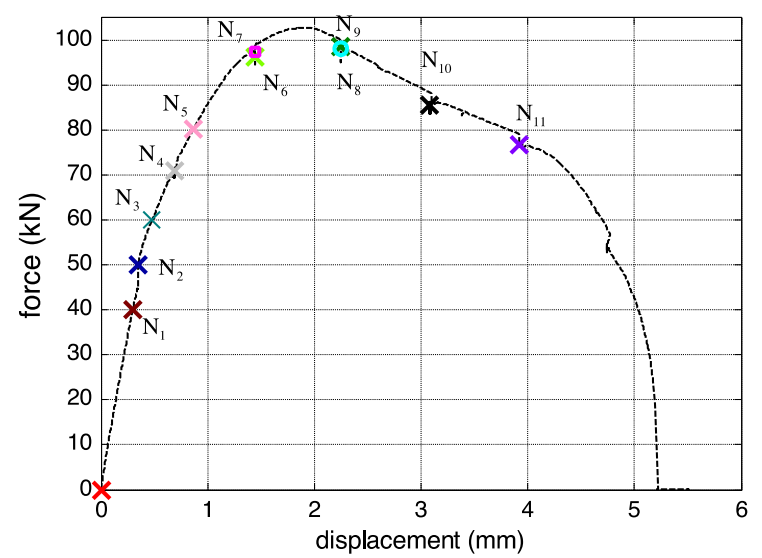

Fig. 12 Evolution of the applied force versus displacement

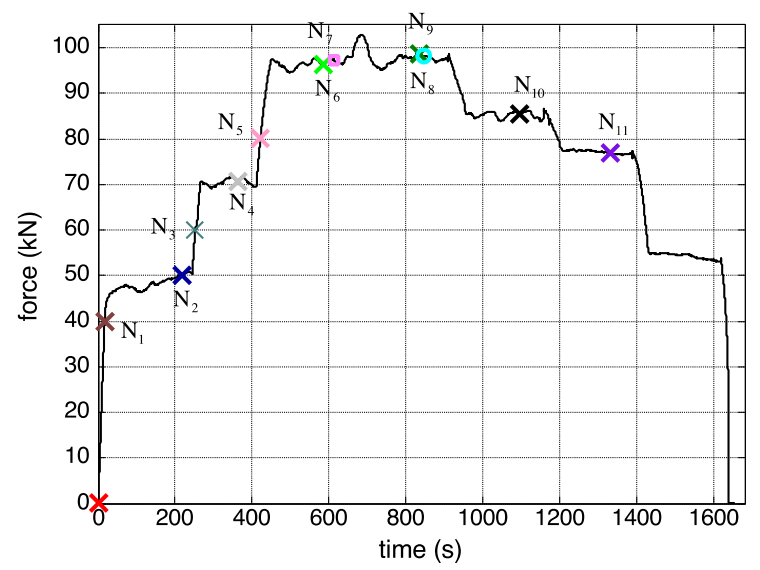

Fig. 13 Evolution of force versus time
Table 1 Values of critical forces and crack tip location

\begin{tabular}{|c|c|c|c|c|c|c|}
\hline \multirow[t]{2}{*}{ Critical $\mathrm{N}_{\mathrm{i}}$} & \multirow{2}{*}{$\begin{array}{l}\text { Force } \\
\mathrm{F}(\mathrm{kN})\end{array}$} & \multirow{2}{*}{$\begin{array}{l}\text { Grip displacement } \\
\mathrm{d}(\mathrm{mm})\end{array}$} & \multirow{2}{*}{$\begin{array}{l}\text { Crack growth } \\
\text { da }(\mathrm{mm})\end{array}$} & \multirow[t]{2}{*}{$\mathrm{dF}(\mathrm{kN})$} & \multicolumn{2}{|c|}{ Crack tip position } \\
\hline & & & & & $\mathrm{X}(\mathrm{mm})$ & $\mathrm{Y}(\mathrm{mm})$ \\
\hline 0 & 0.000 & & 0.000 & & & \\
\hline 1 & 40.000 & 0.297 & 0.000 & 40.000 & 32.333 & 16.947 \\
\hline 2 & 51.110 & 0.35 & 0.150 & 11.110 & 32.233 & 16.835 \\
\hline 3 & 60.000 & 0.477 & 0.082 & 8.890 & 32.178 & 16.774 \\
\hline 4 & 70.950 & 0.686 & 0.046 & 10.950 & 32.179 & 16.728 \\
\hline 5 & 80.160 & 0.864 & 0.018 & 9.210 & 32.162 & 16.721 \\
\hline 6 & 96.340 & 1.441 & 0.071 & 16.180 & 32.097 & 16.692 \\
\hline 7 & 97.350 & 1.441 & 0.422 & 1.010 & 31.899 & 16.320 \\
\hline 8 & 98.680 & 2.246 & 0,815 & 1.330 & 31.240 & 15.840 \\
\hline 9 & 98.260 & 2.245 & 0.963 & -0.420 & 30.600 & 15.120 \\
\hline 10 & 85.500 & 3.083 & 8.867 & -12.720 & 22,000 & 12.960 \\
\hline 11 & 76.880 & 3.922 & 4.585 & -8.620 & 17.440 & 12.480 \\
\hline
\end{tabular}




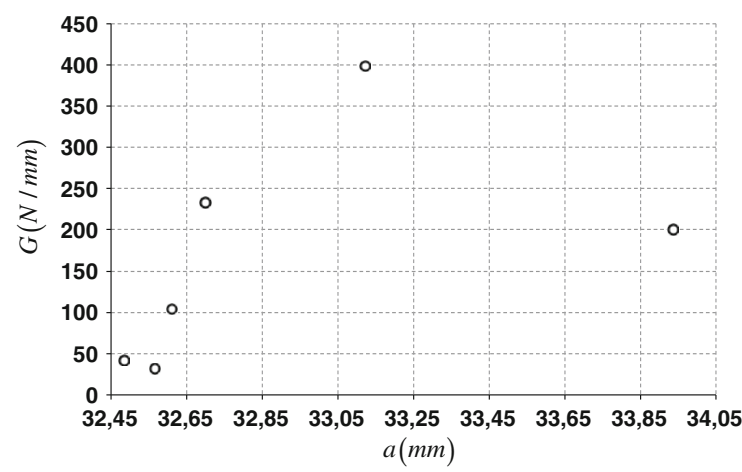

Fig. 14 Experimental elastic energy release rate $G$ versus crack length: compliance method

is given by the following equation

$G_{C}=\frac{F_{C}^{2}}{2 \cdot b} \cdot\left(\frac{\Delta C}{\Delta a}\right)_{d}$

where $C=\frac{\Delta d}{\Delta F_{C}}, F_{C}$ is the critical load inducing a crack propagation length $d a, b$ is the thickness of the specimen (equal to $10 \mathrm{~mm}$ here), and $C$ is the compliance given by Eq. (4). $d$ denotes the displacement of the moving grip. $\Delta F_{C}$ represents the change in force between two consecutives critical points reported in Table 1.

The critical energy release rate is calculated using Eq. (4) and the values reported in Table 1. The points corresponding to the critical numbers $0,1,6,9,10$ and 11 were not taken into account. This reason is the singularity of the compliance formulation (4) in imposed displacements when the crack propagation is too small. Figure 14 shows the evolution of the critical energy release rate versus crack length. For this figure, we note that the evolution of the crack tip lies between 32.50 and $33.95 \mathrm{~mm}$ and that the critical energy release rate increases around $400 \mathrm{~N} / \mathrm{mm}$. The location of the last point ( $G=200 \mathrm{~N} / \mathrm{mm}$, which is lower than $G$ for the preceding loading stages) is justified by the decrease of the energy release rate and the beginning of the material collapse, as shown in Fig. 13.

\subsection{Numerical energy release rate}

The finite element software cast3M has been used in order to implement the $\mathrm{M} \theta$ integral and obtain numerical results. The value for the Young's modulus $E$ and the Poisson's ratio $v$ are those already given above.

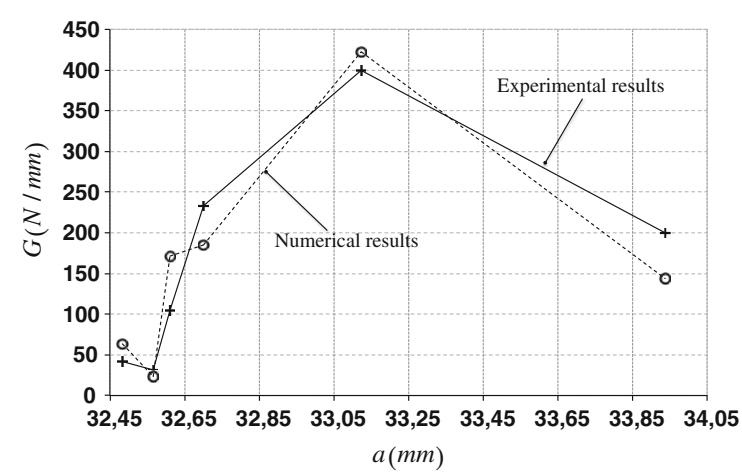

Fig. 15 Comparison of numerical and experimental energy release rate versus crack length

The boundary conditions are those depicted in Fig. 1. Figure 15 shows the comparison between the numerical energy release rate given by the $\mathrm{M} \theta$ integral $\mathrm{Eq}$. (2), and the experimental data obtained using the compliance approach explained above Eq. (4). Numerical and experimental results are in good agreement. The small difference which is observed is due to the fact that identifying the critical force inducing the specific crack length during the crack process is somewhat tricky.

\section{Conclusion}

The grid method was employed here to measure the displacement, strain and local rotation fields near the crack tip of a pre-cracked specimen subjected to a tensile test. It has been shown that these fields are very similar to those obtained with a FE analysis in 2D configuration. The strain and rotation fields were employed to deduce the crack tip location in the images and to measure the crack propagation during the test. The crack tip propagation was also employed to obtain the critical release rate at various load levels using the compliance method. Finally, the $\mathrm{M} \theta$ integral in the crack growth case has been implemented in a finite element software in order to compute the numerical energy release rate. The obtained values have been compared with the experimental data. This illustrates the efficiency of the proposed identification crack tip technique and the relevancy of the numerical approach. In further work, the model proposed in this paper will be generalized to mixed mode crack growth process and deconvolution will be used to enhance the strain maps near the crack tip (Grédiac et al. 2013). 
Open Access This article is distributed under the terms of the Creative Commons Attribution License which permits any use, distribution, and reproduction in any medium, provided the original author(s) and the source are credited.

\section{References}

Atkinson C, Eftaxiopoulos DA (1992) Crack tip stress intensities in viscoelastic anisotropic bimaterials and the use of the Mintegral. Int J Fract 57:61-83

Badulescu C, Grédiac M, Mathias J-D, Roux D (2009a) A procedure for accurate one-dimensional strain measurement using the grid method. Exp Mech 49(6):841-854

Badulescu C, Grédiac M, Mathias J-D (2009b) Investigation of the grid method for accurate in-plane strain measurement. Meas Sci Technol 20(9):1-17

Chalivendra VB (2009) Mixed-mode crack-tip stress fields for orthotropic functionally graded materials. Acta Mech 204:5160. doi:10.1007/s00707-008-0047-1

Chen FMK, Shield RT (1977) Conservation laws in elasticity of J-integral type. J Appl Mech Phys 28:1-22

Destuynder P, Djaoua M, Lescure S (1983) Quelques remarques sur la mécanique de la rupture élastique. J Mech Theory Appl 2:113-135

Dubois F, Moutou Pitti R (2012) Finite element model for crack growth process in concrete bituminous. Adv Eng Softw 44(1):35-43. doi:10.1016/j.advengsoft.2011.05.039

Grédiac M, Toussaint E (2013) Studying the mechanical behaviour of asphalt mixtures with the grid method. Strain 49:1-15

Grédiac M, Sur F, Badulescu C, Mathias J-D (2013) Using deconvolution to improve the metrological performance of the grid method. Opt Lasers Eng 51:716-734

Mathieu F, Hild F, Roux S (2011) Fatigue crack propagation law measured from integrated digital image correlation: the example of Ti35 thin sheets. Procedia Eng 10:1091-1091. doi:10.1016/j.proeng.2011.04.180

McNeill SR, Peters WH, Sutton MA (1987) Estimation of stress intensity factor by digital image correlation. Eng Fract Mech 28:101-112. doi:10.1016/0013-7944(87)90124-X

Méité M, Dubois F, Pop O, Absi J (2013) Mixed mode fracture properties characterization for wood by digital images correlation and finite element method coupling. Eng Fract Mech 105:86-100. doi:10.1016/j.engfracmech.2013.01.008

Méité M, Dubois F, Pop O, Absi J (2013b) Characterization of mixed-mode fracture based on a complementary analysis by means of full-field optical and finite element approaches. Int J Fract 180:41-52. doi:10.1007/s10704-012-9794-z

Molimard J, Surrel Y (2012) Grid method, moiré and deflectometry, in full-field measurements and identification in solid mechanics, Wiley, Grédiac M, Hild, F (eds). ISBN 9781848212947

Moutou Pitti R, Dubois F, Petit C, Sauvat N (2007) Mixed mode fracture separation in viscoelastic orthotropic media: numerical and analytical approach by the $M \theta v$-integral. Int J Fract 145:181-193. doi:10.1007/s10704-007-9111-4
Moutou Pitti R, Dubois F, Petit C et al (2008) A new M integral parameter for mixed mode crack growth in orthotropic viscoelastic material. Eng Fract Mech 75:4450-4465. doi:10. 1016/j.engfracmech.2008.04.021

Moutou Pitti R, Chateauneuf A (2012) Statistical approach and reliability analysis for mixed-mode applied to wood material. Wood Sci Technol 46(6):1099-1112. doi:10.1007/ s00226-011-0462-7

Noether E (1971) Invariant variations problems. Transp Theory Stat Phys 1:183-207

Parks DM (1974) A stiffness derivative finite element technique for determination of crack tip stress intensity factors. Int $\mathrm{J}$ Fract 10:487-502

Parks DM (1977) The virtual crack extension method for nonlinear material behavior. Comput Methods Appl Mech Eng 12:353-364

Piro JL, Grédiac M (2004) Producing and transferring lowspatial-frequency grids for measuring displacement fields with moiré and grid methods. Exp Tech 28(4):23-26

Pop O, Meite M, Dubois F, Absi J (2011) Identification algorithm for fracture parameters by combining DIC and FEM approaches. Int J Fract 170:101-114. doi:10.1007/ s10704-011-9605-y

Réthore J, Gravouil A, Morestin F, Combescure A (2005) Estimation of mixed-mode stress intensity factors using digital image correlation and an interaction integral. Int J Fract 132:65-79

Réthore J, Roux S, Hild F (2008) Noise-robust stress intensity factor determination from kinematic field measurements. Eng Fract Mech 75:3763-3781. doi:10.1016/j.engfracmech.2007. 04.018

Réthoré J, Roux S, Hild F (2010) Mixed-mode crack propagation using a hybrid analytical and extended finite element method. C R Mecanique 338:121-126. doi:10.1016/j.crme.2010.03. 001

Réthoré J, Roux S, Hild F (2012) Optimal and noise-robust extraction of fracture mechanics parameters from kinematic measurements. Eng Fract Mech 78:1827-1845. doi:10.1016/ j.engfracmech.2011.01.012

Rice JR (1968) A path independent integral and the approximate analysis of strain conservation by notches and cracks. J Appl Mech 35:379-385

Suo X, Combescure A (1992) On the application of the Gtheta method and its comparison with de Lorenzi's approach. Nucl Eng Design 135:207-224

Sur F, Grédiac M (2014) Towards deconvolution to enhance the grid method for in-plane strain measurement. (Forthcoming)

Surrel Y (2000) Fringe analysis, in photomechanics. Topics Appl Phys 77, P.K. Rastogi, pp 55-102

Yates JR, Zanganeh M, Tai YH (2010) Quantifying crack tip displacement fields with DIC. Eng Fract Mech 77:2063-2070. doi:10.1016/j.engfracmech.2010.03.025

Zanganeh M, Lopez-Crespo P, Tai YH, Yates JR (2013) Locating the crack tip using displacement field data: a comparative study. Strain 42:102-115 